\title{
Where Birt-Hogg-Dubé meets Cowden Syndrome: mirrored genetic defects in two cases of syndromic oncocytic tumours
}

\author{
Laura Maria Pradella ${ }^{1,4}$, Martin Lang ${ }^{1,4}$, Ivana Kurelac ${ }^{1}$, Elisa Mariani ${ }^{1}$, Flora Guerra ${ }^{1}$, Roberta Zuntini ${ }^{1}$, \\ Giovanni Tallini ${ }^{2}$, Alan MacKay ${ }^{3}$, Jorge S Reis-Filho ${ }^{3}$, Marco Seri ${ }^{1}$, Daniela Turchetti ${ }^{1}$ and \\ Giuseppe Gasparre ${ }^{\star, 1}$
}

Birt-Hogg-Dubè (BHD) is an autosomal dominant syndrome characterised by skin fibrofolliculomas, lung cysts, spontaneous pneumothorax and renal cancer. The association of benign cutaneous lesions and increased cancer risk is also a feature of Cowden Syndrome (CS), an autosomal dominant disease caused by PTEN mutations. BHD and CS patients may develop oncocytomas, rare neoplasias that are phenotypically characterised by a prominent mitochondrial hyperplasia. We here describe the genetic analysis of a parotid and a thyroid oncocytoma, developed by a BHD and a CS patient, respectively. The BHD lesion was shown to maintain the wild-type allele of $F L C N$, while losing one PTEN allele. On the other hand, a double heterozygosity for the same two genes was found to be the only detectable tumorigenic hit in the CS oncocytoma. Both conditions occurred in a context of high chromosomal stability, as highlighted by comparative genomic hybridisation analysis. We conclude that, similarly to PTEN, FLCN may not always follow the classical Two Hits model of tumorigenesis and may hence belong to a class of non-canonical tumour suppressor genes. We hence introduce a role of PTEN/FLCN double heterozygosity in syndromic oncocytic tumorigenesis, suggesting this to be an alternative determinant to pathogenic mitochondrial DNA mutations, which are instead the genetic hallmark of sporadic oncocytic tumours.

European Journal of Human Genetics (2013) 21, 1169-1172; doi:10.1038/ejhg.2013.8; published online 6 February 2013

Keywords: Birt-Hogg-Dubè; Cowden Syndrome; PTEN; FLCN; oncocytoma

\section{INTRODUCTION}

Birt-Hogg-Dubè (BHD) is an autosomal dominantly inherited syndrome characterised by skin fibrofolliculomas, multiple lung cysts, spontaneous pneumothorax and renal cancer (Online Mendelian Inheritance in Man \#135150). It is caused by germline mutations in the folliculin (FLCN) gene (Chr17p11), described to act as a canonical tumour suppressor gene (TSG). ${ }^{1}$ Although the function of FLCN is still unknown, it has been shown to act upstream of mammalian target of rapamycin complex 1 (mTORC1) $)^{2-5}$ and a role in energy/ nutrient-sensing signalling pathways has been suggested. ${ }^{6}$ The association of benign cutaneous lesions and increased cancer risk is also a feature of Cowden Syndrome (CS), an autosomal dominant disease caused by PTEN (phosphatase and tensin homologue deleted on Chromosome 10) mutations and mainly characterised by macrocephaly, mucocutaneous lesions and cancer predisposition. ${ }^{7}$

BHD and CS patients may develop rare type of neoplasms, namely oncocytic tumours. Generally, such tumours originate from epithelial tissues and are phenotypically characterised by prominent mitochondrial hyperplasia, which confers a typical 'swollen', pink eosinophilic appearance to neoplastic cells. ${ }^{8}$ Functionally, sporadic oncocytic tumours are characterised by a respiratory chain derangement due to pathogenic mutations in mitochondrial DNA (mtDNA) genes. The resulting energetic impairment is thought to trigger a compensatory mitochondrial biogenesis ${ }^{9}$ regulated by the peroxisome proliferatoractivated receptor $-\gamma$ coactivator $1 \alpha$ (PGC1 $\alpha) .{ }^{10}$ PGCl $\alpha$ expression is also regulated by mTORC1. The inhibitory function of PTEN on mTORC1 signalling is well known, as PTEN activation results in AKT inhibition, tuberous sclerosis complexes TSC1/TSC2 de-repression and subsequent mTORC1 inhibition. Clinical similarities between BHD and TSC suggested that FLCN and TSC1/TSC2 may function within the same pathway and helped attribute FLCN a regulatory role on mTORC1. However, such a role is yet to be clarified. In fact, evidence from animal models suggest that FLCN may upregulate mTORC1, acting within the same pathway of TSC1/TSC2, albeit in an opposite way. ${ }^{11}$ Another FLCN knockout model, however, supports a role for FLCN in downregulating mTORC1. ${ }^{2,3}$ It has also been put forward that FLCN may both up- or downregulate mTORC1 in a context-dependent manner. ${ }^{4}$ Moreover, FLCN has been shown to negatively correlate with PGC1 $\alpha$ activation, indicating that a decrease of the functional protein may induce mitochondrial biogenesis. ${ }^{12,13}$ We performed a comprehensive genetic analysis of two oncocytic tumours, developed by a BHD and a CS patient, and observed a mirrored genetic background that may account for the development of oncocytic features of syndrome-associated tumours.

${ }^{1}$ Dip. Sc. Mediche e Chirurgiche-DIMEC, U.O. Genetica Medica, Policlinico Universitario S.Orsola-Malpighi, Bologna, Italy; ${ }^{2}$ Dipartimento di Medicina Specialistica, Diagnostica e Sperimentale, Bologna, Italy; ${ }^{3}$ Molecular Pathology Team, The Breakthrough Breast Cancer Research Centre, ICR, London, UK

4These authors contributed equally to this work.

*Correspondence: Dr G Gasparre, Dip. Scienze Mediche e Chirurgiche-DIMEC, U.O. Genetica Medica-Pad.11, Pol.S.Orsola-Malpighi, via Massarenti 9, Bologna 40138, Italy. Tel: + 39 (0)51 2088430; Fax: + 39 (0)51 2088416; E-mail: giuseppe.gasparre@gmail.com

Received 17 September 2012; revised 14 December 2012; accepted 10 January 2013; published online 6 February 2013 


\section{MATERIALS AND METHODS}

See Supplementary Methods.

\section{RESULTS}

We performed an extensive characterisation of a parotid oncocytoma arisen in a BHD patient who showed typical clinical features of BHD, including skin fibrofolliculomas, lung cysts and pneumothorax. $\mathrm{He}$ carried a previously reported germline heterozygous frameshift mutation in exon 5 of FLCN, namely, the c.347dupA (p.Leu117AlafsX16) ${ }^{14}$ (Figure 1a), detected by routine peripheral blood sequencing.

To investigate a possible loss of heterozygosity (LOH) of FLCN, or to find a mutation in the remaining allele, all coding exons were sequenced in the parotid lesion. Such analysis did not show any further alteration and confirmed the persistence of the heterozygous mutation, suggesting no loss of the wild-type FLCN allele in the neoplastic tissue. Additional investigation by real-time PCR-based copy number assay and multiplex ligation-dependent probe amplification (MLPA) corroborated the persistence of the wild-type allele in the tumour cells (Figure 1b).

We hence screened the tumour for mtDNA mutations, hallmarks of sporadic oncocytomas. Sequencing of the whole mtDNA merely revealed common polymorphisms and ruled out somatic mtDNA mutations as the main causative genetic hit of oncocytic tumorigenesis in this sample.

To investigate the presence of potential oncogenic hits in nuclear DNA genes, the mutational hot spots of the most commonly altered oncogenes (PIK3CA, BRAF, CTNNB1, KRAS, HRAS, AKT, KIT,
PIK3R1 and ERBB2) were screened and no pathogenic mutations were detected. Two TSGs frequently involved in tumorigenesis, PTEN and TP53, were also sequenced and no mutations were detected. However, as no heterozygous polymorphism was observed along the PTEN sequence, we investigated a possible $\mathrm{LOH}$ and thereby demonstrated the complete deletion of one PTEN allele by real-time PCR analysis in the tumour-derived DNA, compared to the peritumoural tissue-derived DNA of the BHD patient (Figure 1c).

Based on the finding of a co-occurrence of PTEN and FLCN alterations in this patient, we returned to a CS patient in which we previously reported a germline heterozygous PTEN deletion and who developed a thyroid oncocytoma, among other typical CS features such as macrocephaly, dysplastic gangliocytoma of the cerebellum, papillary thyroid carcinoma, papillomatous lesions on the skin and at the mouth angles. ${ }^{15}$ These lesions were screened for FLCN point mutations, yet none were found. However, the oncocytic neoplasm resulted to be homozygous for a known single-nucleotide polymorphism (SNP; rs8065832), which was heterozygous in the other pathogenic lesions (Figure 1d). This feature suggested the occurrence of a gene deletion, which was confirmed by a real-time PCR-based copy number assay (Figure 1e), in which tumour-derived DNA was compared to that extracted from the peripheral blood of the patient.

To understand whether the described genetic alterations were part of widespread chromosomal rearrangements in the tumours, array CGH (aCGH) analysis was performed. ${ }^{16}$ The oncocytic parotid tumour of the BHD patient showed a deletion of $633 \mathrm{kbs}$ on Chr10, spanning PTEN, which was further defined by microsatellite

\section{a}

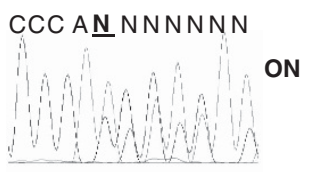

CCCA G C T C T T C

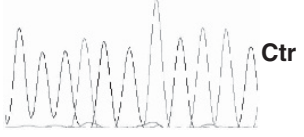

d

G GTAGG $\underline{\mathbf{Y}} \mathrm{GGCA}$ b

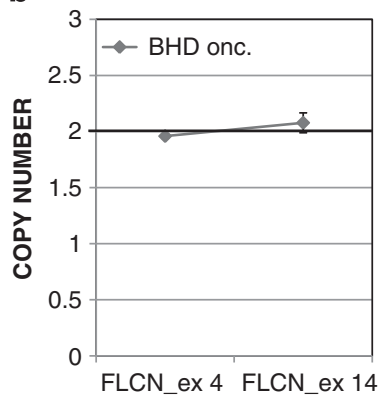

C

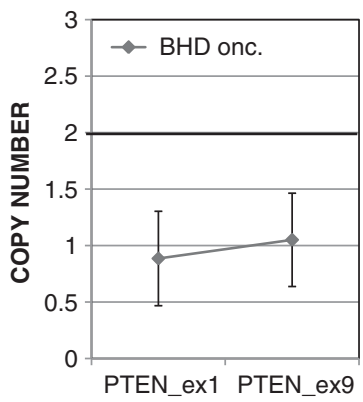

e

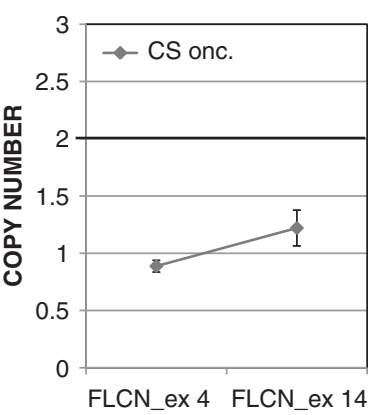

Figure 1 Genetic findings in BHD and CS cases. (a) FLCN sequence showing heterozygosity of c.347dupA mutation in the oncocytic tumour tissue (ON) of BHD patient compared to a control sequence obtained on a healthy subject (Ctr). (b) Real-time PCR copy number assay performed on exon 4 and exon 14 of FLCN confirming persistence of two alleles in the BHD oncocytoma. (c) Validation of LOH of PTEN in BHD oncocytoma by real-time PCR. (d) Electropherogram of FLCN showing SNP rs 8065832 to be heterozygous in the blood and papillary thyroid carcinoma (PTC) of the CS patient, whereas being homozygous in the respective oncocytoma (ON). (e) Real-time PCR copy number assay confirming LOH of FLCN in the oncocytoma developed by the CS patient. Mutated positions are indicated in bold and underlined. 
markers (10q23.33-10q26.13). This tumour did not show other major genomic aberrations (Figure 2a).

The molecular karyotype of the CS oncocytoma confirmed the presence of a deletion on Chr10q and showed two large duplications on Chr5 and Chr7, partial loss of Chr17p, as well as additional smaller losses and gains (Figure 2b).

Last, in order to verify if a double heterozygosity of FLCN and PTEN may be a mechanism substituting for the presence of mtDNA mutations, we analysed PTEN and FLCN in 22 sporadic mtDNAmutated oncocytic lesions, which included 12 thyroid and 10 parotid gland tumours, for which DNA extracted from tumour and nontumour tissue was available. The PTEN/FLCN analysis was based on a workflow aimed at optimising available DNA and costs, to first rule out either gene heterozygosity (Supplementary Figure 1). No point mutations and no deletions in FLCN and/or PTEN were detected, suggesting that PTEN and FLCN were not altered in the presence of mtDNA mutations and that instead their double heterozygosity may be a peculiarity of oncocytic tumours associated to Mendelian cancer syndromes only.

\section{DISCUSSION}

We here describe two cases of BHD- and CS-associated parotid and thyroid oncocytic tumours, respectively. These neoplasms were shown to harbour mirrored genetic defects in PTEN and FLCN (summarised in Figure 2C). PTEN is known to behave as a haploinsufficient TSG that exerts its function on tumour initiation and progression in a dosedependent manner. ${ }^{17}$ A small reduction of PTEN expression is sufficient to trigger tumour formation in mice, and heterozygosity of PTEN is generally maintained in malignancies developed by CS patients. ${ }^{18}$

FLCN, on the other hand, has been thus far considered a canonical TSG, the two alleles of which are inactivated in tumour cells by LOH or somatic mutations. ${ }^{19}$ Somatic 'second hit' FLCN mutations or $\mathrm{LOH}$ on chromosome $17 \mathrm{p}$ are, in fact, indentified in the vast majority of BHD-associated renal tumours. Conversely, in BHD skin lesions the wild-type allele is generally retained. ${ }^{20}$ In the minority of tumour cases in which a wild-type FLCN allele is retained, it may not be ruled out that the second tumorigenic hit may occur at a different locus.

The same effect in triggering tumorigenesis as complete loss of function of FLCN may therefore be reached by inactivating one allele of FLCN together with one allele of a different TSG that would similarly maintain one wild-type allele in the somatic lesion.

In this context FLCN would not necessarily behave as a canonical TSG, strengthening its similarity to PTEN. Further studies are warranted to understand whether the FLCN/PTEN double heterozygosity is sufficient to trigger tumorigenesis, and they are ongoing in our laboratory.

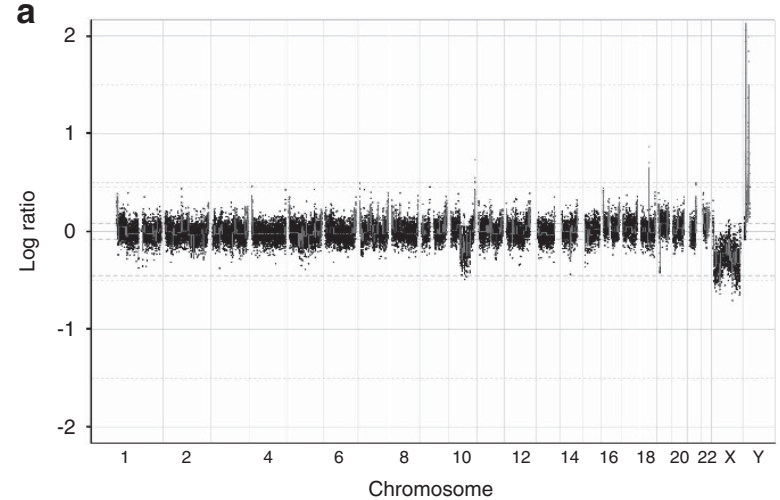

C

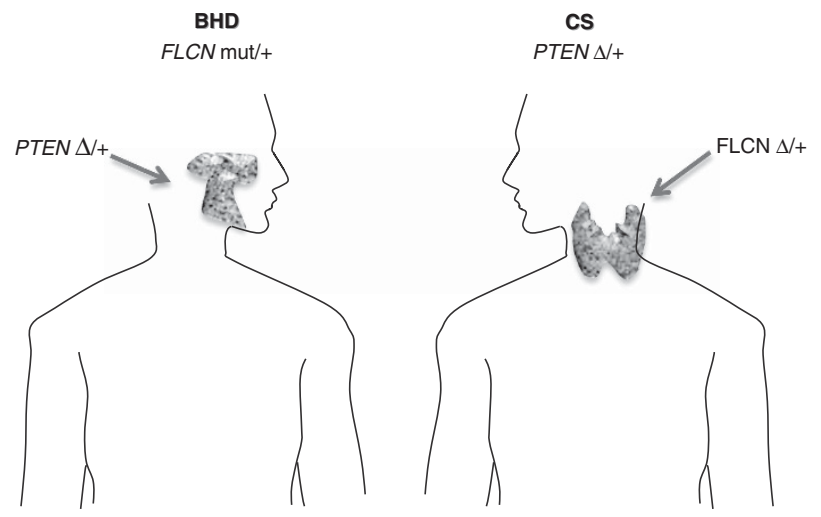

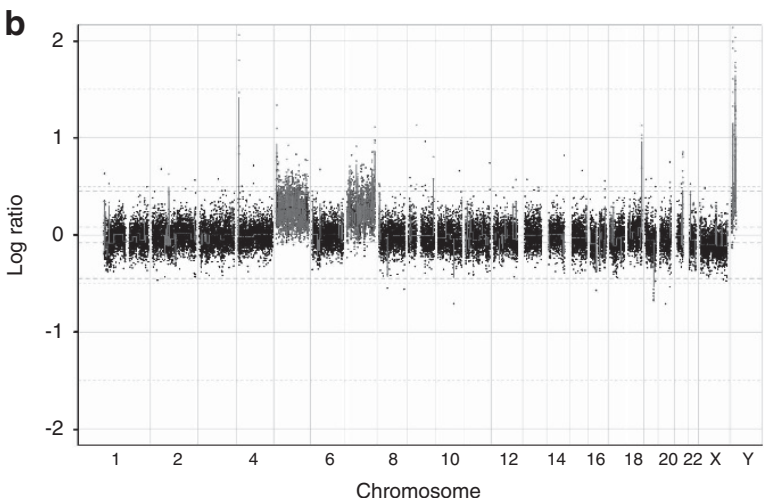

d

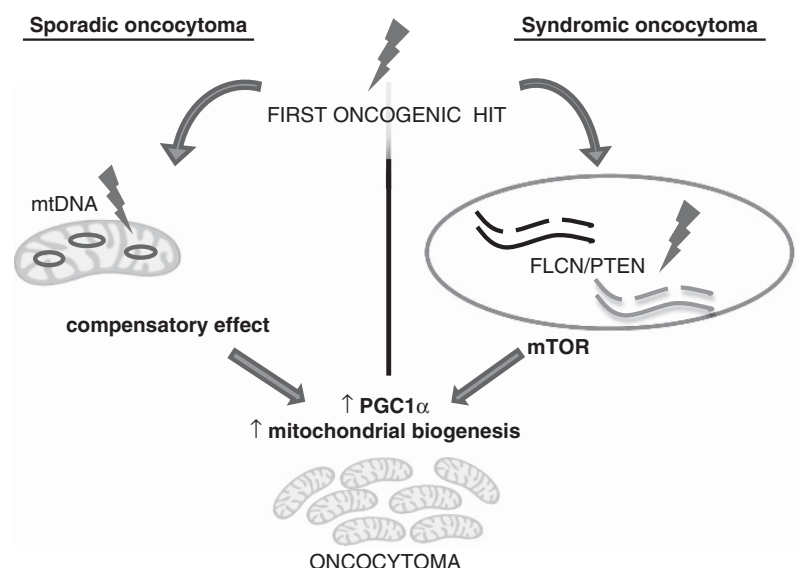

Figure 2 Array comparative genomic hybridisation of BHD (a) and CS (b) oncocytic neoplasms. Large deletion of Chr10q in the BHD sample (a) as well as a small deletion of Chr10q, a partial loss of Chr17p and large duplications of Chr5 and Chr7 in CS sample (b) can be observed. (c) Schematic representation of the mirrored genetic profile in parotid and thyroid oncocytomas of BHD and CS patients. Germline mutations as well as somatic hits in PTEN and FLCN are shown. (d) Alternative pathways involved in the formation of sporadic and syndromic oncocytomas. Upon a first tumorigenic hit, either mtDNA mutations or FLCN/PTEN double heterozygosity, increase in PGCl $\alpha$ transcription and subsequent increase of mitochondrial biogenesis, the main feature of oncocytic cancers, would occur. 
It appears clear that the requirement for TSGs that do not follow the classical Two-Hits model for a second somatic event to prompt tumorigenesis is fulfilled in the mirrored cases we report (Figure 2c). The analysis of oncogenes and TSGs commonly mutated in cancer revealed, in fact, the occurrence of a mono-allelic loss of PTEN in the BHD parotid oncocytoma. This finding raised the hypothesis that PTEN may cooperate with FLCN, specifically in oncocytic tumorigenesis. Support to this theory is provided by the detection of a heterozygous deletion of FLCN in the CS thyroid oncocytic cancer. Interestingly, $\mathrm{LOH}$ of FLCN was excluded in the other lesions of the CS patient analysed, reinforcing the idea that the association between the germline PTEN and the somatic FLCN deletion is specifically related to the oncocytic phenotype. This association may not be coincidental as FFPE-specific aCGH did not show additional chromosomal aberrations in the BHD sample, and few were shown in the CS sample. Such mirrored genetic defects of BHD- and CS-associated oncocytic neoplasms suggest that the two syndromes may be related and that FLCN and PTEN probably converge in a common pathway, the deregulation of which may concur to oncocytic tumorigenesis in an alternative mode of action with respect to pathogenic mtDNA mutations (Figure 2d). In fact, it is generally acknowledged that the genetic hallmarks of all types of oncocytic sporadic tumours are disassembling mtDNA mutations, which are thought to induce mitochondrial hyperplasia as a compensatory response to the respiratory chain dysfunction. ${ }^{9}$ The energetic impairment most likely induces a retrograde signal to the nucleus, which activates the expression of different regulators of mitochondrial biogenesis, the most important of which being PGC1 $\alpha .{ }^{10}$ However, both BHD- and CS-associated oncocytic tumours here described lacked acquired, tumour-specific mtDNA mutations, ruling out this driving mechanism for oncocytic transformation.

PTEN and FLCN have been suggested to act as regulators of mTORC1, which in turn has been shown to induce PGC1 $\alpha$ transcription. ${ }^{21}$ Interestingly, FLCN has been recently demonstrated to act as a negative regulator of PGC1 $\alpha$ expression levels both in conditional FLCN knockout mice and in cell models, ${ }^{13}$ reinforcing the hypothesis of an involvement in oncocytic transformation also in a context different from BHD, such as CS. Alterations in gene expression levels due to genetic changes of the two non-canonical TSGs FLCN and PTEN, which act on converging pathways, may thus either cause oncocytic transformation after the occurrence of an oncogenic triggering event, or account for a primary transformation along with the appearance of an oncocytic phenotype.

In conclusion, we here propose that the causative gene of BHD, FLCN, may not necessarily follow the classical Two-Hits model of tumorigenesis and may belong to a class of non-canonical TSGs of which PTEN is a well-known member. Moreover, we introduce for the first time a role of PTEN/FLCN double heterozygosity in hereditary oncocytic transformation and suggest this to represent an alternative mechanism to that induced by pathogenic mtDNA mutations.

\section{CONFLICT OF INTEREST}

The authors declare no conflict of interest.

\section{ACKNOWLEDGEMENTS}

This work was supported by Associazione Italiana Ricerca sul Cancro (AIRC) grant IG8810; by grant FIRB 'Futuro in Ricerca' 2008; and by a grant from Fondazione Umberto Veronesi to G.G.; L.M.P. is supported by a grant from the Myrovlytis Trust (London, UK); M.L. is supported by an annual outbound fellowship from AIRC/FIRC 'Fellowship For Abroad 2011'; I.K. is supported by a triennial fellowship from AIRC/FIRC 'Borromeo'; F.G. is supported by a biennial fellowship from AIRC/FIRC 'Maria Antonietta Carluccio'. J.S.R.-F. and A.M. are funded by Breakthrough Breast Cancer.

1 Nickerson ML, Warren MB, Toro JR et al: Mutations in a novel gene lead to kidney tumors, lung wall defects, and benign tumors of the hair follicle in patients with the Birt-Hogg-Dube syndrome. Cancer Cell 2002; 2: 157-164.

2 Baba M, Furihata M, Hong SB et al: Kidney-targeted Birt-Hogg-Dube gene inactivation in a mouse model: Erk1/2 and Akt-mTOR activation, cell hyperproliferation, and polycystic kidneys. J Natl Cancer Inst 2008; 100: 140-154.

3 Hasumi Y, Baba M, Ajima R et al: Homozygous loss of BHD causes early embryonic lethality and kidney tumor development with activation of mTORC1 and mTORC2. Proc Natl Acad Sci USA 2009; 106: 18722-18727.

4 Hudon V, Sabourin S, Dydensborg $A B$ et al: Renal tumour suppressor function of the Birt-Hogg-Dube syndrome gene product folliculin. J Med Genet 2010; 47: $182-189$.

5 Chen J, Futami K, Petillo D et al: Deficiency of FLCN in mouse kidney led to development of polycystic kidneys and renal neoplasia. PLoS One 2008; 3 e3581.

6 Baba M, Hong SB, Sharma N et al: Folliculin encoded by the BHD gene interacts with a binding protein, FNIP1, and AMPK, and is involved in AMPK and mTOR signaling. Proc Natl Acad Sci USA 2006; 103: 15552-15557.

7 Hollander MC, Blumenthal GM, Dennis PA: PTEN loss in the continuum of common cancers, rare syndromes and mouse models. Nat Rev Cancer 2011; 11: 289-301.

8 Tallini G: Oncocytic tumours. Virchows Arch 1998; 433: 5-12.

9 Gasparre G, Romeo G, Rugolo M, Porcelli AM: Learning from oncocytic tumors: Why choose inefficient mitochondria? Biochim Biophys Acta 2011; 1807: 633-642.

10 Liang H, Ward WF: PGC-1alpha: a key regulator of energy metabolism. Adv Physiol Educ 2006; 30: 145-151.

11 Hartman TR, Nicolas E, Klein-Szanto A et al: The role of the Birt-Hogg-Dube protein in mTOR activation and renal tumorigenesis. Oncogene 2009; 28: 1594-1604.

12 Klomp JA, Petillo D, Niemi NM et al: Birt-Hogg-Dube renal tumors are genetically distinct from other renal neoplasias and are associated with up-regulation of mitochondrial gene expression. BMC Med Genomics 2010; 3: 59.

13 Hasumi H, Baba M, Hasumi Y et al: Regulation of Mitochondrial Oxidative Metabolism by Tumor Suppressor FLCN. J Natl Cancer Inst 2012; 104: 1750-1764.

14 Toro JR, Wei MH, Glenn GM et al: BHD mutations, clinical and molecular genetic investigations of Birt-Hogg-Dube syndrome: a new series of 50 families and a review of published reports. J Med Genet 2008; 45: 321-331.

15 Pradella LM, Zuntini R, Magini $P$ et al: Two distinct thyroid tumours in a patient with Cowden syndrome carrying both a 10q23 and a mitochondrial DNA germline deletion. J Med Genet 2011; 48: 779-782.

16 Kurelac I, Mackay A, Lambros MB et al: Somatic complex I disruptive mitochondrial DNA mutations are modifiers of tumorigenesis that correlate with low genomic instability in pituitary adenomas. Hum Mol Genet 2012; 22: 226-238.

17 Carracedo A, Alimonti A, Pandolfi PP: PTEN level in tumor suppression: how much is too little? Cancer Res 2011; 71: 629-633.

18 Marsh DJ, Dahia PL, Coulon V et al: Allelic imbalance, including deletion of PTEN/ $\mathrm{MMACl}$, at the Cowden disease locus on 10q22-23, in hamartomas from patients with Cowden syndrome and germline PTEN mutation. Genes Chromosomes Cancer 1998; 21: 61-69.

19 Vocke CD, Yang Y, Pavlovich CP et al: High frequency of somatic frameshift BHD gene mutations in Birt-Hogg-Dube-associated renal tumors. J Natl Cancer Inst 2005; 97: 931-935.

20 Schmidt LS: Birt-Hogg-Dube syndrome: from gene discovery to molecularly targeted therapies. Fam Cancer 2012; e-pub ahead of print 30 October 2012; PMID:23108783.

21 Cunningham JT, Rodgers JT, Arlow DH, Vazquez F, Mootha VK, Puigserver P: mTOR controls mitochondrial oxidative function through a YY1-PGC-1alpha transcriptional complex. Nature 2007; 450: 736-740.

Supplementary Information accompanies the paper on European Journal of Human Genetics website (http://www.nature.com/ejhg) 